\title{
Optimal Green Hybrid Attacks in Secure IoT
}

\author{
Bhawna Ahuja, Student Member, IEEE, Deepak Mishra, Member, IEEE, and Ranjan Bose, Senior Member, IEEE
}

\begin{abstract}
In pursuance to understand the behavior of a potential green hybrid attacker in secure internet-of-things (IoT), this letter investigates optimal energy utilization from the attacker's viewpoint. Specifically, we propose a novel framework for optimizing the efficacy of a hybrid attacker, possessing capability to both eavesdrop and jam, while considering the underlying energy consumption. In particular, we maximize the attacker energy efficiency (AEE) in secure IoT by deriving the analytical solutions for jointly global-optimal attacking mode, eavesdropping rate, and jamming power. Numerical results, validating analytical claims, reveal that the proposed green design can offer about $45 \%$ improvement in average AEE over the relevant benchmarks.
\end{abstract}

Index Terms-Physical layer security, energy efficiency, global optimization, eavesdropping, jamming, power control.

\section{INTRODUCTION}

Physical layer security (PLS) is a promising approach for investigating the security threats in resource-constrained setups like internet of things (IoT) [1], especially with an intelligent hybrid attacker capable of carrying both eavesdropping and jamming [2]. Along with the security, energy efficiency (EE) has also become important from both user and attacker's perspective [3]. This has led to a timely demand for investigating a green hybrid attacker which may be a potential threat for practical IoT applications like smart home and smart city.

Recent studies have shown interest on optimizing the EE of jamming attacker [3], [4]. Whereas optimal attacking strategies for hybrid attacker are designed in [5], [6] to achieve maximum degradation in the secrecy rate, however EE aspect has been neglected. Further, decoding-based energy-expenditures in eavesdropping and practical circuit-level consumption during jamming have been ignored while considering energy consumption at attacker [3]-[7]. In contrast, for the IoT applications using low power wireless communication technologies e.g. Bluetooth Low Energy (BLE), ZigBee, LoRa, SigFox etc., active power at receiver becomes non-negligible in comparison to that at transmitter [8]. This can have a significant effect on power consumption strategies, hence deciding whether to eavesdrop or jam, becomes a nontrivial task for the attacker.

We address this challenge by proposing novel energyaware attacking strategies for hybrid attackers using theoretical analyses of PLS in secure IoT. Major contributions include: (i) First-time characterization of the secrecy rate degradation arising out of underlying joint energy-aware eavesdropping and jamming. The key aspect is to consider energy consumption during both attacker modes and propose a new

B. Ahuja and R. Bose are with the Bharti School of Telecommunication Technology and Management, IIT Delhi,110016 New Delhi. R. Bose is also affiliated with IIIT Delhi. e-mail: (bhawna.ahuja,rbose)@iitd.ac.in

D. Mishra is with the Department of School of Electrical Engineering and Telecommunications, UNSW Sydney, NSW 2052, Australia (e-mail: d.mishra@unsw.edu.au). metric called attacker energy efficiency (AEE) that can fairly model the combined degradation effect. (ii) Derivation of a semi-closed form expression for joint global-optimal attacking mode, eavesdropping rate and jamming power in jamming mode yielding maximal AEE. (iii) Practically-motivated numerical investigation for validating the proposed analysis, that includes tight analytical approximation for global-optimal jamming power, and quantifying the achievable AEE gains over relevant benchmarks.

\section{Novel Green Hybrid AtTACKer Model}

\section{A. Network Topology and Channel Model}

We consider secure data transmission in an IoT system, wherein an information source $\mathcal{S}$ communicates with a legitimate user $\mathcal{U}$ using power $P_{\mathcal{S}}$ in the presence of an attacker $\mathcal{A}$. Here, $\mathcal{A}$ is a malicious, but smart IoT node possessing the dual capability of eavesdropping and jamming, though having a limited energy budget $P_{m}$. $\mathcal{A}$ works in a half-duplex mode [2] and while eavesdropping, it turns on its receiver, and attempts to tap the legitimate transmissions of $\mathcal{S}$. Alternatively, when $\mathcal{A}$ acts as a jammer, it sends a jamming signal at power $P_{\mathcal{J}}$ to impair the legitimate receptions at $\mathcal{U}$. The corresponding channel gains over $\mathcal{S}-\mathcal{U}, \mathcal{S}-\mathcal{A}$ and $\mathcal{A}-\mathcal{U}$ links are respectively denoted by $\mathrm{g}_{\mathcal{S U}}, \mathrm{g}_{\mathcal{S A}}$ and $\mathrm{g}_{\mathcal{A U}}$. These links are assumed to be perfectly known at $\mathcal{A}$ [7] for each coherence block of unit duration.

\section{B. Degraded Secrecy Rate}

The respective signal-to-noise ratios (SNRs) for links $\mathcal{S}$ $\mathcal{U}$ and $\mathcal{S}-\mathcal{A}$ are given by $\gamma_{\mathcal{S U}}=\frac{P_{\mathcal{S}} \mathrm{g}_{\mathcal{S U}}}{\sigma^{2}}$ and $\gamma_{\mathcal{S A}}=\frac{P_{\mathcal{S}} \mathrm{g}_{\mathcal{S} \mathcal{A}}}{\sigma^{2}}$ where $\sigma^{2}$ is the received noise power at $\mathcal{U}$ and $\mathcal{A}$. Accordingly, respective communication rates or spectral efficiencies $R_{\mathcal{U}}$ and $R_{\mathcal{A}}$ over links $\mathcal{S}-\mathcal{U}$ and $\mathcal{S}-\mathcal{A}$ in (bps/Hz) are calculated as $R_{\mathcal{U}}=\log _{2}\left(1+\gamma_{\mathcal{S U}}\right)$ and $R_{\mathcal{A}}=\log _{2}\left(1+\gamma_{\mathcal{S A}}\right)$. Now, the secrecy rate $R_{\mathcal{E}}$ for $\mathcal{S}-\mathcal{U}$ communication under eavesdropping attack by $\mathcal{A}[1]$ is :

$$
R_{\mathcal{E}}=\max \left(0, R_{\mathcal{U}}-R_{\mathcal{A}}\right)
$$

Similarly under $\mathcal{A}$ 's jamming mode, secrecy rate $R_{\mathcal{J}}$ [2] is:

$R_{\mathcal{J}}=\log _{2}\left(1+\frac{P_{\mathcal{S}} \mathrm{g}_{\mathcal{S U}}}{P_{\mathcal{J}} \mathrm{g}_{\mathcal{A U}}+\sigma^{2}}\right)=\log _{2}\left(1+\frac{\gamma_{\mathcal{S U}}}{1+\gamma_{\mathcal{A U}}}\right)$,

where $\gamma_{\mathcal{A U}}=\frac{P_{\mathcal{J}} \mathrm{g}_{\mathcal{A U}}}{\sigma^{2}}$ represents the SNR of $\mathcal{A}-\mathcal{U}$ link.

Now, as $\mathcal{A}$ intends to maximize deterioration to the secrecy rate of $\mathcal{U}$, we express this deterioration to $\mathcal{U}$ 's secure performance via degraded secrecy rate. This metric is quantified as difference of achievable rate during $\mathcal{S}-\mathcal{U}$ communication in absence of $\mathcal{A}$ and the corresponding secrecy rate $R_{\mathcal{E}}$ or $R_{\mathcal{J}}$ in presence of $\mathcal{A}$. Hence, respective degraded secrecy rate $R_{\mathrm{DE}}$ and $R_{\mathrm{D} \mathcal{J}}$ for eavesdropping and jamming are defined as:

$$
R_{\mathrm{DE}}=R_{\mathcal{U}}-R_{\mathcal{E}}=\min \left(R_{\mathcal{A}}, R_{\mathcal{U}}\right)
$$




$$
R_{\mathrm{D} \mathcal{J}}=R_{\mathcal{U}}-R_{\mathcal{J}}=\log _{2}\left(\frac{\left(1+\gamma_{\mathcal{S U}}\right)\left(1+\gamma_{\mathcal{A U}}\right)}{1+\gamma_{\mathcal{S U}}+\gamma_{\mathcal{A U}}}\right)
$$

\section{Consumption Model for Eavesdropping and Jamming}

As in eavesdropping, $\mathcal{A}$ has to receive and decode the signal, we have followed the commonly used model for computing power consumption at receiver [9]-[11], and references therein. For a unit block duration of $1 \mathrm{~s}$, the energy or power consumption at $\mathcal{A}$ in receiving mode is given by: $P_{\mathrm{fr}}+\rho_{\mathrm{d}} r_{\mathcal{A}}$. Here, $P_{\mathrm{fr}}$ is the static consumption required for receiving at $\mathcal{A}, \rho_{\mathrm{d}}$ represents the dynamic unit rate decoding power consumption and $r_{\mathcal{A}}$ represents the decoding rate of $\mathcal{A}$. It may be noted here the decoding rate $r_{\mathcal{A}}$ chosen at $\mathcal{A}$ has to satisfy the constraint $r_{\mathcal{A}} \leq R_{\mathcal{A}}$, because $R_{\mathcal{A}}$ is the maximum rate over $\mathcal{S}-\mathcal{A}$ link as available for eavesdropping. Similarly, as a jammer, $\mathcal{A}$ transmits a jamming signal at power $P_{\mathcal{J}}$, adopting conventional power consumption model for transmitter [9], [12]. Therefore, the power consumption in jamming mode is modelled as: $P_{\mathrm{ft}}+\left(P_{\mathcal{J}} / \nu\right)$. Here, $P_{\mathrm{ft}}$ is static power consumption at transmitting circuitry of $\mathcal{A}$ and $\nu$ is efficiency of power amplifier at $\mathcal{A}$.

\section{Objective Formulation}

We introduce a new performance metric called Attacker Energy Efficiency (AEE) defined as a ratio of the degraded secrecy rate to the total power consumption at $\mathcal{A}$ in achieving it. It is worth to mention that being the decided decoding rate at $\mathcal{A}, r_{\mathcal{A}}$ reflects the effective eavesdropping, henceforth may also be known as eavesdropping rate. Noting that degradation in secrecy rate caused by eavesdropping is upper bounded by $R_{\mathcal{U}}, r_{\mathcal{A}}$ actually characterise the effective degraded secrecy rate under eavesdropping mode which is eventually defined as $\min \left(r_{\mathcal{A}}, R_{\mathcal{U}}\right)$. It is upper bounded by $R_{\mathrm{DE}}$ given by (3) due to constraint $r_{\mathcal{A}} \leq R_{\mathcal{A}}$. Now AEE $\eta_{\mathcal{E}}\left\{r_{\mathcal{A}}\right\}$ for eavesdropping, and $\eta_{\mathcal{J}}\left\{P_{\mathcal{J}}\right\}$ for jamming using (4) are respectively given as:

$$
\eta_{\mathcal{E}}\left\{r_{\mathcal{A}}\right\}=\frac{\min \left(r_{\mathcal{A}}, R_{\mathcal{U}}\right)}{P_{\mathrm{fr}}+\rho_{\mathrm{d}} r_{\mathcal{A}}}, \quad \eta_{\mathcal{J}}\left\{P_{\mathcal{J}}\right\}=\frac{R_{\mathrm{D} \mathcal{J}}}{P_{\mathrm{ft}}+\left(P_{\mathcal{J}} / \nu\right)} .
$$

For incorporating overall degradation in secrecy rate due to both eavesdropping and jamming modes of $\mathcal{A}$, AEE $\eta\left\{\alpha, r_{\mathcal{A}}, P_{\mathcal{J}}\right\}$ can be defined as a ratio of weighted sum of degraded secrecy rate for underlying attacking modes to the weighted sum of their respective power consumption.

$\eta\left\{\alpha, r_{\mathcal{A}}, P_{\mathcal{J}}\right\}=\frac{\alpha \min \left(r_{\mathcal{A}}, R_{\mathcal{U}}\right)+(1-\alpha) R_{\mathrm{D} \mathcal{J}}}{\alpha\left(P_{\mathrm{fr}}+\rho_{\mathrm{d}} r_{\mathcal{A}}\right)+(1-\alpha)\left(P_{\mathrm{ft}}+\left(P_{\mathcal{J}} / \nu\right)\right)},(6)$

where $\alpha$ is the fraction of unit block duration assigned for eavesdropping and $(1-\alpha)$ is allocated for jamming.

We aim to maximise AEE $\eta\left\{\alpha, r_{\mathcal{A}}, P_{\mathcal{J}}\right\}$ by jointly optimising $\alpha, r_{\mathcal{A}}, P_{\mathcal{J}}$ as in optimization problem (P0), subject to eavesdropping rate constraints $(C 1-C 2)$, jamming power constraints $(C 3-C 4)$, normalization constraints on $\alpha(C 5-C 6)$, and total power constraint $(C 7)$ at $\mathcal{A}$, is:

$$
\begin{aligned}
& (\mathrm{P} 0): \max _{r_{\mathcal{A}}, P_{\mathcal{J}}, \alpha} \eta\left\{\alpha, r_{\mathcal{A}}, P_{\mathcal{J}}\right\}, \quad \text { subject to: } \\
& C 1: r_{\mathcal{A}} \leq R_{\mathcal{A}}, C 2: r_{\mathcal{A}} \geq 0, C 3: P_{\mathcal{J}} \leq P_{\mathcal{J} m}, \\
& C 4: P_{\mathcal{J}} \geq 0, \quad C 5: \alpha \geq 0, \quad C 6: \alpha \leq 1, \\
& C 7: \alpha\left(P_{\mathrm{fr}}+\rho_{\mathrm{d}} r_{\mathcal{A}}\right)+(1-\alpha)\left(P_{\mathrm{ft}}+\left(P_{\mathcal{J}} / \nu\right)\right) \leq P_{m} .
\end{aligned}
$$

Here, $P_{\mathcal{J} m}$ in $C 3$ is maximum allowed jamming power. Next, we present jointly global-optimal solution for (P0).

\section{Proposed Solution Methodology}

The original problem (P0) is a nonlinear non-convex optimization problem due to non-concavity of objective function $\eta\left\{\alpha, r_{\mathcal{A}}, P_{\mathcal{J}}\right\}$ in $\alpha, r_{\mathcal{A}}$ and $P_{\mathcal{J}}$. So, we first propose an equivalent transformation strategy involving decomposition of (P0) into three sub-problems and then obtain the jointly globaloptimal solution by using outcomes of these 3 sub-problems.

\section{A. Optimal Mode Selection}

The sub-problem (P1) to obtain optimal $\alpha$ that maximizes $\eta\{\alpha\}$ for a given $r_{\mathcal{A}}$ and $P_{\mathcal{J}}$, is defined as:

$(\mathrm{P} 1): \max _{\alpha} \eta\{\alpha\}, \quad$ subject to: $C 5, C 6, C 7$.

The solution of (P1) is presented by Lemma 1 as given below.

Lemma 1: The global-optimal value of $\alpha$ is either 1 or 0 .

Proof: Depending on input parameters, $\eta\{\alpha\}$ will either be increasing or decreasing function of $\alpha$ as shown next by taking first order derivative of $\eta\{\alpha\}$ with respect to $\alpha$ :

$$
\begin{aligned}
\frac{\partial \eta\{\alpha\}}{\partial \alpha}= & \frac{\left(P_{\mathrm{fr}}+\rho_{\mathrm{d}} r_{\mathcal{A}}\right)\left(P_{\mathrm{ft}}+\left(P_{\mathcal{J}} / \nu\right)\right)\left(\frac{\min \left(r_{\mathcal{A}}, R_{\mathcal{U}}\right)}{P_{\mathrm{fr}}+\rho_{\mathrm{d}} r_{\mathcal{A}}}-\frac{R_{\mathrm{D} \mathcal{J}}}{P_{\mathrm{ft}}+\left(P_{\mathcal{J}} / \nu\right)}\right)}{\left(\alpha\left(P_{\mathrm{fr}}+\rho_{\mathrm{d}} r_{\mathcal{A}}\right)+(1-\alpha)\left(P_{\mathrm{ft}}+\left(P_{\mathcal{J}} / \nu\right)\right)^{2}\right.} \\
& \stackrel{a}{=} \frac{\left(P_{\mathrm{fr}}+\rho_{\mathrm{d}} r_{\mathcal{A}}\right)\left(P_{\mathrm{ft}}+\left(P_{\mathcal{J}} / \nu\right)\right)\left(\eta_{\mathcal{E}}\left\{r_{\mathcal{A}}\right\}-\eta_{\mathcal{J}}\left\{P_{\mathcal{J}}\right\}\right)}{\left(\alpha P_{\mathrm{fr}}+\rho_{\mathrm{d}} r_{\mathcal{A}}+(1-\alpha) P_{\mathrm{ft}}+\left(P_{\mathcal{J}} / \nu\right)\right)^{2}}
\end{aligned}
$$

where $a$ is obtained using (5). Being $\frac{\partial \eta\{\alpha\}}{\partial \alpha}>0$ and $\frac{\partial \eta\{\alpha\}}{\partial \alpha} \leq$ 0 respectively for $\eta_{\mathcal{E}}\left\{r_{\mathcal{A}}\right\}>\eta_{\mathcal{J}}\left\{P_{\mathcal{J}}\right\}$ and $\eta_{\mathcal{E}}\left\{r_{\mathcal{A}}\right\} \leq$ $\eta_{\mathcal{J}}\left\{P_{\mathcal{J}}\right\}$, it is observed that $\eta\{\alpha\}$ is an increasing function of $\alpha$ in the former case and decreasing function otherwise. Since $\eta_{\mathcal{E}}\left\{r_{\mathcal{A}}\right\}$ and $\eta_{\mathcal{J}}\left\{P_{\mathcal{J}}\right\}$ do not vary with $\alpha$, both can be easily compared to find optimal solution existing at extreme points of $\alpha$, i.e., 1 or 0 . In other words, assigning only one attacking mode either eavesdropping or jamming is optimal as follows:

$$
\alpha^{\star}=\left\{\begin{array}{lll}
1 & \text { (Eavesdropping), } & \eta_{\mathcal{E}}\left\{r_{\mathcal{A}}\right\}>\eta_{\mathcal{J}}\left\{P_{\mathcal{J}}\right\} \\
0 & \text { (Jamming), } & \text { otherwise }
\end{array}\right.
$$

where $\alpha^{\star}$ is optimal solution of (P1).

Remark 1: Noting the two possible values of $\alpha^{\star}$, objective function $\eta\left\{\alpha, r_{\mathcal{A}}, P_{\mathcal{J}}\right\}$ of problem (P0) equivalently reduces to $\eta_{\mathcal{E}}\left\{r_{\mathcal{A}}\right\}$ for $\alpha^{\star}=1$ or $\eta_{\mathcal{J}}\left\{P_{\mathcal{J}}\right\}$ for $\alpha^{\star}=0$. It shows that $r_{\mathcal{A}}$ and $P_{\mathcal{J}}$ are decoupled from each other at optimal value of $\alpha$ that enables each variable to be individually optimised.

\section{B. Optimal Eavesdropping Rate}

For $\alpha=1$, (P0) is reduced to (P2) where objective function $\eta_{\mathcal{E}}\left\{r_{\mathcal{A}}\right\}$ represents AEE for eavesdropping only.

$$
(\mathrm{P} 2): \max _{r_{\mathcal{A}}} \eta_{\mathcal{E}}\left\{r_{\mathcal{A}}\right\}, \quad \text { subject to: } C 1, C 2, C 7 \text {. }
$$

Its solution is provided via Lemma 2.

Lemma 2: The global-optimal solution of (P2) is

$$
r_{\mathcal{A}}^{\star} \triangleq \min \left(R_{\mathrm{DE}}, \frac{P_{m}-P_{\mathrm{fr}}}{\rho_{\mathrm{d}}}\right) .
$$

where $\rho_{\mathrm{d}}$ is measured in $\mathrm{W} / \mathrm{bps} / \mathrm{Hz}$.

Proof: As observed from (5), $\eta_{\mathcal{E}}\left\{r_{\mathcal{A}}\right\}$ is a decreasing function of $r_{\mathcal{A}}$ for $r_{\mathcal{A}}>R_{\mathcal{U}}$. On the other hand, as $\frac{\partial \eta_{\mathcal{E}}\left\{r_{\mathcal{A}}\right\}}{\partial r_{\mathcal{A}}}=$ $\frac{P_{\mathrm{fr}}}{\left(P_{\mathrm{fr}}+\rho_{\mathrm{d}} r_{\mathcal{A}}\right)^{2}}>0, \forall r_{\mathcal{A}} \in\left[0, R_{\mathcal{A}}\right]$ we can observe that $\eta_{\mathcal{E}}\left\{r_{\mathcal{A}}\right\}$ is strictly increasing function of $r_{\mathcal{A}}$ for $r_{\mathcal{A}} \leq R_{\mathcal{U}}$. Therefore, 
the global-optimal solution of (P2) can be obtained by solving the boundary constraints on $r_{\mathcal{A}}$ as defined via $C 1, C 2$ and $C 7$ for $\alpha=1$. On solving them and using (3), we obtain (9).

\section{Optimal Jamming Power}

For $\alpha=0$, (P0) gets transformed to (P3), where $\eta_{\mathcal{J}}$ is to be maximised with $\mathcal{A}$ being in jamming mode.

(P3) : $\max _{P_{\mathcal{J}}} \eta_{\mathcal{J}}\left\{P_{\mathcal{J}}\right\}, \quad$ subject to: $C 3, C 4, C 7$.

Below, we present the key result leading to global-optimal solution of $(\mathrm{P} 3)$.

Lemma 3: $\eta_{\mathcal{J}}\left\{P_{\mathcal{J}}\right\}$ is a psuedo-concave function of $P_{\mathcal{J}}$.

Proof: Using (4) and (5), it is found that $R_{\mathrm{D} \mathcal{J}}$, the numerator of $\eta_{\mathcal{J}}\left\{P_{\mathcal{J}}\right\}$, is a concave function of $P_{\mathcal{J}}$ as $\frac{d^{2} R_{\mathrm{D} \mathcal{J}}}{d P_{\mathcal{J}}^{2}}=$ $-\frac{\left(\mathrm{g}_{\mathcal{S U}}\right)^{2} \mathrm{~g}_{\mathcal{S} \mathcal{A}} P_{\mathcal{S}}\left(2 \mathrm{~g}_{\mathcal{A U}} P_{\mathcal{J}}+\mathrm{g}_{\mathcal{S U}} P_{\mathcal{S}}+2 \sigma^{2}\right)}{\log (2)\left(\mathrm{g}_{\mathcal{A U}} P_{\mathcal{J}}+\sigma^{2}\right)^{2}\left(\mathrm{~g}_{\mathcal{A U}} P_{\mathcal{J}}+\mathrm{g}_{\mathcal{S U}} P_{\mathcal{S}}+\sigma^{2}\right)^{2}}<0, \forall$ $P_{\mathcal{J}} \in\left[0, P_{\mathcal{J} m}\right]$. Next, since the denominator $P_{\mathrm{ft}}+\left(P_{\mathcal{J}} / \nu\right)$ of $\eta_{\mathcal{J}}\left\{P_{\mathcal{J}}\right\}$ is an affine transformation of $P_{\mathcal{J}}$ and the ratio of concave function to positive affine function is a pseudoconcave function [13, (Table 5.5)], we notice that $\eta_{\mathcal{J}}\left\{P_{\mathcal{J}}\right\}$ is pseudo-concave in $P_{\mathcal{J}}$. This completes the proof.

Lemma 3 implies that the global-optimal solution of (P3) as denoted by $P_{\mathcal{J}}^{\star}$ is obtained by solving $\frac{d \eta_{\mathcal{J}}\left\{P_{\mathcal{J}}\right\}}{d P_{\mathcal{J}}}=0$. Since, it is not possible to obtain the explicit analytic solution for $P_{\mathcal{J}}^{\star}$, we apply golden section (GS) search within lower bound $P^{l}=0$ and upper bound $P^{u}=\min \left(P_{\mathcal{J} m}, \nu\left(P_{m}-P_{\mathrm{ft}}\right)\right)$ as obtained using $C 3, C 4$ and $C 7$ with $\alpha=0$. Next, we analyse the complexity of global-optimal solution of (P3) obtained numerically. In addition, we also introduce an approximate closed-form solution for $P_{\mathcal{J}}^{\star}$ to get further analytical insights.

1) Complexity Analysis: $P_{\mathcal{J}}^{\star}$ can be found efficiently in few iterations $N$, which can be calculated noting that the GS search terminates when $\left(P^{u}-P^{l}\right)(0.618)^{N} \leq \epsilon$ where $\epsilon$ is the acceptable tolerance. Thus, the order of complexity for the proposed solution, in Big O notation, is $O\left(\log \left(\frac{\min \left(P_{\mathcal{J} m}, \nu\left(P_{m}-P_{\mathrm{ft}}\right)\right)}{\epsilon}\right)\right)$.

2) Proposed Analytical Approximation: Here, we propose an approximate closed-form solution for $P_{\mathcal{J}}^{\star}$ for the asymptotic case with $\gamma_{\mathcal{S U}} \gg 1$ and $\gamma_{\mathcal{A U}} \gg 1$ along with $P_{\mathcal{J}} \gg P_{\mathrm{ft}}$ [7]. Under these approximations, using (4) and (5), objective function of $(\mathrm{P} 3)$ can be reduced to:

$\eta_{\mathcal{J}}\left\{P_{\mathcal{J}}\right\} \approx \frac{\nu \log _{2}(\phi)}{P_{\mathcal{J}}}$ with $\phi=\frac{P_{\mathcal{S}} \mathrm{g}_{\mathcal{S U}} P_{\mathcal{J}} \mathrm{g}_{\mathcal{A U}}}{\left(P_{\mathcal{S}} \mathrm{g}_{\mathcal{S U}}+P_{\mathcal{J}} \mathrm{g}_{\mathcal{A U}}\right) \sigma^{2}}$.

Hereby, analytical approximation for $P_{\mathcal{J}}^{\star}$ is obtained by first

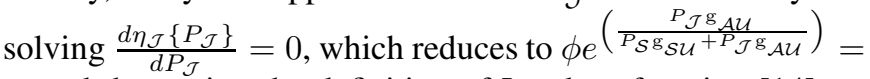
$e$, and then using the definition of Lambert function [14] as:

$$
\widehat{P_{\mathcal{J}}^{\star}} \triangleq \frac{P_{\mathcal{S}} \mathrm{g}_{\mathcal{S U}} \mathrm{W}\left(\frac{e}{\gamma_{\mathcal{S U}}}\right)}{\mathrm{g}_{\mathcal{A U}}\left[1-\mathrm{W}\left(\frac{e}{\gamma_{\mathcal{S U}}}\right)\right]}
$$

\section{Jointly Global-optimal Solution $\left(\alpha^{*}, r_{\mathcal{A}}^{*}, P_{\mathcal{J}}^{*}\right)$ for $(P 0)$}

As explained in Lemma 1 and Remark 1, optimal solutions of $r_{\mathcal{A}}$ and $P_{\mathcal{J}}$ are found using their decoupling for both optimal value of $\alpha$ through (P2) and (P3) respectively. Lemma 2 and 3 further prove the global-optimality of the solutions for $r_{\mathcal{A}}$ and $P_{\mathcal{J}}$. Finally, we select the optimal setting of $\alpha$ out of 1 or 0 that gives the higher AEE between $\eta_{\mathcal{E}}\left\{r_{\mathcal{A}}^{\star}\right\}$ and

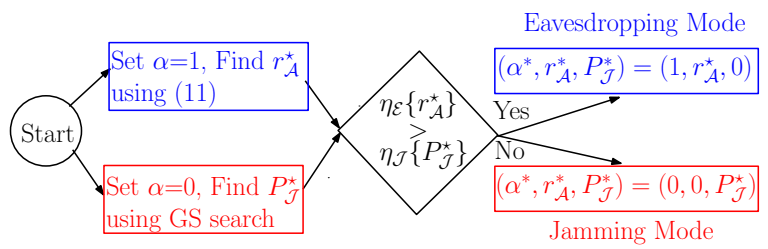

Fig. 1. Graphical description for solution methodology.

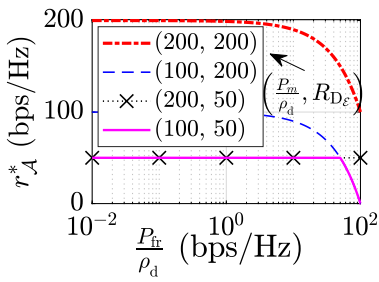

(a) Performance of $r_{\mathcal{A}}^{\star}$

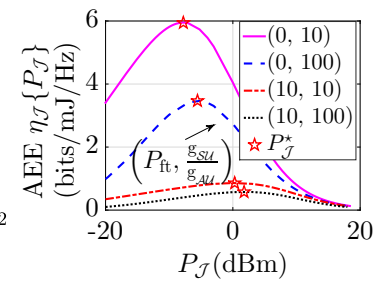

(b) Nature of AEE $\eta_{\mathcal{J}}$ in $P_{\mathcal{J}}$.
Fig. 2. Analysis validation and insights on optimal $r_{\mathcal{A}}$ and $P_{\mathcal{J}}$.

$\eta_{\mathcal{J}}\left\{P_{\mathcal{J}}^{\star}\right\}$. This global-optimal value of $\alpha$ is obtained using (8) while setting $r_{\mathcal{A}}=r_{\mathcal{A}}^{\star}$ and $P_{\mathcal{J}}=P_{\mathcal{J}}^{\star}$ for joint optimisation. Summarising the solution methodology, as also depicted by Fig. 11 the jointly global-optimal solution of (P0) is:

$$
\left(\alpha^{*}, r_{\mathcal{A}}^{*}, P_{\mathcal{J}}^{*}\right)= \begin{cases}\left(1, r_{\mathcal{A}}^{\star}, 0\right) & \eta_{\mathcal{E}}\left\{r_{\mathcal{A}}^{\star}\right\}>\eta_{\mathcal{J}}\left\{P_{\mathcal{J}}^{\star}\right\} \\ \left(0,0, P_{\mathcal{J}}^{\star}\right) & \text { otherwise. }\end{cases}
$$

As $\alpha^{*}=1$ implies only eavesdropping, therefore, $P_{\mathcal{J}}^{*}=0$. Similarly $r_{\mathcal{A}}^{*}=0$ for $\alpha^{*}=0$ since only jamming is performed.

\section{Numerical Results}

The default simulation parameters are: $P_{\mathcal{S}}=10 \mathrm{dBm}$, $P_{\mathcal{J} m}=13 \mathrm{dBm}, P_{m}=13 \mathrm{dBm}, \mathrm{g}_{\mathcal{S U}}=-60 \mathrm{~dB}$, $\mathrm{g}_{\mathcal{S A}}=\mathrm{g}_{\mathcal{A U}}=-70 \mathrm{~dB}, \sigma^{2}=-100 \mathrm{dBm}, \nu=0.7$, $P_{\mathrm{ft}}=P_{\mathrm{fr}}=-0.33 \mathrm{dBm}, \rho_{\mathrm{d}}=-10.33 \mathrm{dBm} / \mathrm{bps} / \mathrm{Hz}$ as the decoding power consumption per unit rate at $\mathcal{A}$ considering Mica2 mote and CC-1000 transceiver to represent low power IoT system [9], [10] and references therein.

First, through Fig. 2(a), we explore the optimal eavesdropping performance by investigating the nature of $r_{\mathcal{A}}^{\star}$ for different values of $R_{\mathrm{DE}}$ and $\frac{P_{m}}{\rho_{\mathrm{d}}}$ in bps/Hz by varying $\frac{P_{\mathrm{fr}}}{\rho_{\mathrm{d}}}$. The impact of different parameters can be categorized into two cases: (i) $R_{\mathrm{DE}} \geq \frac{P_{m}}{\rho_{\mathrm{d}}}$, (ii) $R_{\mathrm{DE}}<\frac{P_{m}}{\rho_{\mathrm{d}}}$. For case (i), $r_{\mathcal{A}}^{\star}$ follows increasing trend with $P_{m}$ and decreasing with $P_{\mathrm{fr}}$ for a given $\rho_{\mathrm{d}}$. A significant drop is observed in $r_{\mathcal{A}}^{\star}$ beyond $\frac{P_{\mathrm{fr}}}{\rho_{\mathrm{d}}}=10$ for the typical values, because higher values of $P_{\text {fr }}$ relative to $\rho_{\mathrm{d}}$ help to reduce $r_{\mathcal{A}}^{\star}$ significantly. For case (ii), $r_{\mathcal{A}}^{\star}$ becomes independent of $P_{m}$ and depends on $P_{\mathrm{fr}}$ for a given $\rho_{\mathrm{d}}$ only for $\frac{P_{\mathrm{fr}}}{\rho_{\mathrm{d}}}>\frac{P_{m}}{\rho_{\mathrm{d}}}-R_{\mathrm{D} \mathcal{E}}$. Otherwise $r_{\mathcal{A}}^{\star}$ remains equal to $R_{\mathrm{D} \mathcal{E}}$ due to boundary constraints. For instance, with $\frac{P_{m}}{\rho_{\mathrm{d}}}=100$ and $R_{\mathrm{DE}}=50$ in Fig. 2 $(\mathrm{a}), r_{\mathcal{A}}^{\star}$ remains equal to $R_{\mathrm{DE}}$ till $\frac{P_{\mathrm{fr}}}{\rho_{\mathrm{d}}} \leq 50$. Later on, it decreases with $P_{\mathrm{fr}}$ for given $\rho_{\mathrm{d}}$.

Next, via Fig 2 (b), we investigate the optimal jamming and validate the pseudo-concavity of AEE $\eta_{\mathcal{J}}\left\{P_{\mathcal{J}}\right\}$ in $P_{\mathcal{J}}$ for different values of $P_{\mathrm{ft}}$ in $\mathrm{dBm}$ and channel gains ratio. Here, $\mathrm{g}_{\mathcal{S U}}$ is kept fixed and only $\mathrm{g}_{\mathcal{A U}}$ is varied. We note that $\eta_{\mathcal{J}}\left\{P_{\mathcal{J}}\right\}$ decreases with increased $P_{\mathrm{ft}}$ because the latter leads to higher 


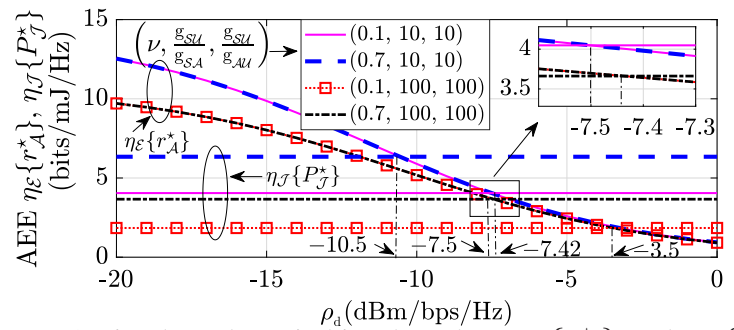

Fig. 3. Optimal mode switching based on $\eta_{\mathcal{E}}\left\{r_{\mathcal{A}}^{\star}\right\}$ and $\eta_{\mathcal{J}}\left\{P_{\mathcal{J}}^{\star}\right\}$.

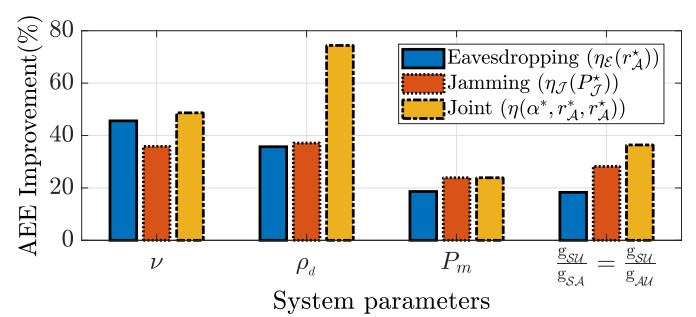

Fig. 4. Performance gain of proposed optimization over benchmark.

power consumption. Also, the underlying $P_{\mathcal{J}}^{\star}$ moves closer to $P_{\mathcal{J} m}$ for further enhancement of secrecy degradation owing to its reduced impact on power consumption. Similarly, $\eta_{\mathcal{J}}\left\{P_{\mathcal{J}}\right\}$ decreases with decreased $\mathrm{g}_{\mathcal{A U}}$ due to reduced degraded secrecy rate and $P_{\mathcal{J}}^{\star}$ increases to strengthen the $\mathcal{A}-\mathcal{U}$ link. Results also show that static power consumption has a significant impact in addition to channel parameters on $\eta_{\mathcal{J}}\left\{P_{\mathcal{J}}^{\star}\right\}$ and hence, the former cannot be ignored unlike existing literature did.

Now we would like to provide novel insights on the optimal mode switching at $\mathcal{A}$ depending on power consumption and channel parameters. We can note from (12) that $\eta_{\mathcal{E}}\left\{r_{\mathcal{A}}^{\star}\right\}$ and $\eta_{\mathcal{J}}\left\{P_{\mathcal{J}}^{\star}\right\}$ are deciding factors for optimal mode switching, and joint optimal AEE can be calculated as: $\eta\left(\alpha^{*}, r_{\mathcal{A}}^{*}, P_{\mathcal{J}}^{*}\right)=\max \left(\eta_{\mathcal{E}}\left\{r_{\mathcal{A}}^{\star}\right\}, \eta_{\mathcal{J}}\left\{P_{\mathcal{J}}^{\star}\right\}\right)$. Thus, $\eta_{\mathcal{E}}\left\{r_{\mathcal{A}}^{\star}\right\}$ and $\eta_{\mathcal{J}}\left\{P_{\mathcal{J}}^{\star}\right\}$ are plotted in Fig. 3 for different values of channel gain ratio, $\nu$ and $\rho_{\mathrm{d}}$. We observe that there exists a switching threshold value of $\rho_{\mathrm{d}}$, below which $\eta_{\mathcal{E}}\left\{r_{\mathcal{A}}^{\star}\right\}$ is greater than $\eta_{\mathcal{J}}\left\{P_{\mathcal{J}}^{\star}\right\}$ resulting in eavesdropping mode as optimal one. Otherwise, for $P_{d}$ values higher than this threshold, jamming is selected as the optimal mode. Results show that for a lower efficiency jammer with $\nu=0.1$, the channel conditions $\left\{\frac{\mathrm{g}_{\mathcal{S U}}}{\mathrm{g}_{\mathcal{S A}}}=\frac{\mathrm{g}_{\mathcal{S U}}}{\mathrm{g}_{\mathcal{A U}}}\right\}=\{10,100\}$ lead to eavesdropping being the optimal mode for $\rho_{\mathrm{d}}<\{-7.5,-3.5\} \mathrm{dBm}$, respectively. However, for an efficient jammer with $\nu=0.7$, this switching threshold value decreases to $\{-10.5,-7.42\} \mathrm{dBm}$ for $\left\{\frac{\mathrm{g}_{\mathcal{S U}}}{\mathrm{g}_{\mathcal{S A}}}=\right.$ $\left.\frac{\mathrm{g}_{\mathcal{S U}}}{\mathrm{g}_{\mathcal{A} \mathcal{U}}}\right\}=\{10,100\}$, respectively. Thus, lower $\mathcal{A}$ 's channel gains $\left(\mathrm{g}_{\mathcal{S} \mathcal{A}}, \mathrm{g}_{\mathcal{A U}}\right)$ favor eavesdropping, whereas higher $\rho_{\mathrm{d}}$ and $\nu$ values favor jamming.

Finally, in Fig. 4, we compare the average AEE performance of three optimization schemes i) optimal eavesdropping (P2) ii) optimal jamming (P3), and iii) joint optimization (P0) against a fixed benchmark scheme with $\alpha=0.5, P_{\mathcal{J}}=0$ $\mathrm{dBm}$ and $r_{\mathcal{A}}=\min \left(\log _{2}\left(1+\gamma_{\mathcal{S A}}\right), \frac{P_{m}-P_{\mathrm{fi}}}{\rho_{\mathrm{d}}}\right)$ which is maximum possible eavesdropping rate. The average percentage (\%) improvement is calculated by first analyzing \% gain in optimal AEE for different values of each parameter $\left(\nu, \rho_{\mathrm{d}}\right.$, $\left.P_{m}, \frac{\mathrm{g}_{\mathcal{S U}}}{\mathrm{g}_{\mathcal{S A}}}, \frac{\mathrm{g}_{\mathcal{S U}}}{\mathrm{g}_{\mathcal{A U}}}\right)$ in its acceptable range and then taking mean over these respective gain values. The corresponding average $\%$ improvement in AEE are plotted for different parameters by varying $\nu$ from $10 \%$ to $90 \%, \rho_{\mathrm{d}}$ from $-20 \mathrm{dBm} / \mathrm{bit}$ to 0
$\mathrm{dBm} / \mathrm{bit}, P_{m}$ from $0 \mathrm{dBm}$ to $13 \mathrm{dBm}, \frac{\mathrm{g}_{\mathcal{S U}}}{\mathrm{g}_{\mathcal{S A}}}$ and $\frac{\mathrm{g}_{\mathcal{S U}}}{\mathrm{g}_{\mathcal{A U}}}$ from 1 to 1000 . The average $\%$ improvement as provided by optimal eavesdropping, jamming and joint schemes are $29.5 \%, 31.5 \%$, $45 \%$ respectively. It is to be noted that with $P_{\mathcal{J}}>0 \mathrm{dBm}$ in fixed benchmark scheme, the average percentage gain in AEE provided by proposed optimization schemes would have been even more higher. Thus, the joint optimization provides a significant improvement in AEE over benchmark scheme, and in general, the optimal jamming is a better semi-adaptive scheme as compared to optimal eavesdropping.

\section{CONCLUding REMARKS}

We have derived the jointly global-optimal attacking mode selection and resource allocation for maximizing the AEE. Numerical investigations validate the key metric characterizing the non-trivial mode switching based solution methodology (c.f. Fig. 1) for solving the otherwise non-convex problem. Significant improvement of $45 \%$ in average AEE is achieved using proposed joint optimization over the benchmark.

\section{REFERENCES}

[1] Y. Wu, A. Khisti, C. Xiao, G. Caire, K. Wong, and X. Gao, "A survey of physical layer security techniques for $5 \mathrm{G}$ wireless networks and challenges ahead," IEEE J. Sel. Areas Commun., vol. 36, no. 4, pp. 679-695, Apr. 2018.

[2] H. Chen, X. Tao, N. Li, Y. Hou, J. Xu, and Z. Han, "Secrecy performance analysis for hybrid wiretapping systems using random matrix theory," IEEE Trans. Wireless Commun., vol. 18, no. 2, pp. 1101-1114, Feb. 2019.

[3] M. A. Maleki Sadr, M. Ahmadian-Attari, R. Amiri, and V. V. Sabegh, "Worst-case jamming attack and optimum defense strategy in cooperative relay networks," IEEE Control Syst. Lett., vol. 3, no. 1, pp. 7-12, Jan. 2019.

[4] Z. Yang, P. Cheng, and J. Chen, "Learning-based jamming attack against low-duty-cycle networks," IEEE Trans. Depend. Sec. Comput., vol. 14, no. 6, pp. 650-663, Nov. 2017.

[5] L. Xiao, C. Xie, M. Min, and W. Zhuang, "User-centric view of unmanned aerial vehicle transmission against smart attacks," IEEE Trans. Veh. Technol., vol. 67, no. 4, pp. 3420-3430, Apr. 2018.

[6] A. Garnaev, M. Baykal-Gursoy, and H. V. Poor, "A game theoretic analysis of secret and reliable communication with active and passive adversarial modes," IEEE Trans. Wireless Commun., vol. 15, no. 3, pp. 2155-2163, Mar. 2016.

[7] B. Li, Y. Yao, H. Zhang, and Y. Lv, "Energy efficiency of proactive cooperative eavesdropping over multiple suspicious communication links," IEEE Trans. Veh. Technol., vol. 68, no. 1, pp. 420-430, Jan. 2019.

[8] A. Mammela and A. Anttonen, "Why will computing power need particular attention in future wireless devices?" IEEE Circ. Syst. Mag., vol. 17, no. 1, pp. 12-26, Firstquarter 2017.

[9] D. Mishra, S. De, and D. Krishnaswamy, "Dilemma at RF energy harvesting relay: Downlink energy relaying or uplink information transfer?" IEEE Trans. Wireless Commun., vol. 16, no. 8, pp. 4939-4955, Aug. 2017.

[10] D. Mishra and S. De, "Effects of practical rechargeability constraints on perpetual RF harvesting sensor network operation," IEEE Access, vol. 4, pp. 750-765, Mar. 2016.

[11] P. R. Gautam, S. Kumar, A. Verma, T. Rashid, and A. Kumar, "Energyefficient localization of sensor nodes in WSNs using beacons from rotating directional antenna," IEEE Trans. Ind. Informat., pp. 1-9, Apr. 2019, early access.

[12] D. Chen, W. Yang, J. Hu, Y. Cai, and X. Tang, "Energy-efficient secure transmission design for the Internet of Things with an untrusted relay," IEEE Access, vol. 6, pp. $11862-11$ 870, 2018.

[13] M. Avriel, E. Diewerth, S. Schaible, and I. Zang, Generalized concavity. Philadelphia, PA, USA: SIAM, vol. 63, 2010.

[14] E. W. Weisstein, "Lambert W-Function," From MathWorld - A Wolfram Web Resource, accessed Sep. 25, 2018. [Online]. Available: http://mathworld.wolfram.com/LambertW-Function.html 